This manuscript has been submitted for publication in Renewable Energy. Please note that it has not been peer-reviewed and has not yet been accepted for publication. Subsequent versions of this manuscript may have different content. If accepted, the final version of this manuscript will be available via the 'Peerreviewed Publication DOI' link on the right-hand side of this webpage. Please feel free to contact any of the authors; we welcome feedback. 


\title{
A copula-based assessment of renewable energy droughts across Europe
}

\author{
Noelia Otero ${ }^{\mathrm{a}}$, Olivia Martius ${ }^{\mathrm{a}}$, Sam Allen ${ }^{\mathrm{b}}$, Hannah Bloomfield ${ }^{\mathrm{c}, \mathrm{d}}$, Bettina \\ Schaefli $^{\mathrm{a}}$ \\ ${ }^{a}$ Oeschger Centre for Climate Change Research, University of Bern, Bern, Switzerland \\ ${ }^{b}$ Institute of Mathematical Statistics and Actuarial Science, University of Bern, Bern, \\ Switzerland \\ ${ }^{c}$ Department of Meteorology, University of Reading, Reading, United Kingdom \\ ${ }^{d}$ Department of Geographical Sciences, University of Bristol, Bristol, UK
}

\begin{abstract}
Meeting carbon-reduction targets will require thorough consideration of climate variability and climate change due to the increasing share of climatesensitive renewable energy sources (RES). One of the main concerns arises from situations of low renewable production and high demand, which can hinder the power system. We analysed energy droughts, defined as periods of low energy production (wind plus solar generation) or high residual load (demand minus production), in terms of two main properties: duration and severity. We estimated the joint return periods associated with energy droughts of residual load and power production. We showed that moderate winter energy droughts of both low renewable production and high residual load occur every half a year, while summer events occur every 3.6 and 2.4 years (on average). As expected, the occurrence of energy droughts tends to decrease with the degree of the severity of the energy drought, and moderate and extreme energy droughts showed longer return period for most countries. In general, we found a large variability across Europe in summer, with some countries (e.g. Italy) being more sensitive to energy droughts. Our results highlight the relevance of sharing RES during prolonged periods of low production and high demand.
\end{abstract}

Keywords: Energy drought, wind power, solar power, frequency, copula,

Email address: noelia.otero@giub.unibe.ch (Noelia Otero)

Preprint submitted to Renewable energy

December 1, 2021 
return period, duration, severity

\section{Introduction}

A rapid decarbonization of the energy system is required to mitigate the effects of climate change (Brucker et al. 2014$)$. Europe is expected to reach a climate-neutral economy with large reductions in green house emissions to at

5 least $80 \%$ below 1990 levels by 2050 (European Climate Foundation, 2010). This ambitious plan towards a low-carbon power system is influenced by a changing climate, not only on the production side but also on the demand side as heating and cooling patterns are changing as a result of rising temperatures (e.g. Cronin et al., 2018: Wenz et al., 2017). Balancing variable energy supply and demand might become a major concern in the design of renewable power systems, due to a strong sensitivity to weather and climate variability. In particular, wind and solar power installed capacities have rapidly grown over the past years EEA (2017), 2017) and they are expected to be important contributors to the European renewable power system. However, their fluctuating nature represents a challenge for renewable energy production as both sources are directly dependent on weather conditions with a high spatio-temporal variability (von Bremen 2010, Francois, 2016; Jurasz et al., 2021).

As a result of the intermittency that characterizes the renewable energy sources (RES), balancing the RES generation and energy consumption is a key concern, since electricity demand must be continuously matched by electricity supply to avoid blackouts (von Bremen, 2010). The residual load (or net load) is the imbalance between RES and the energy demand (sometimes called load), and is defined as the difference between the energy demand and the energy production von Bremen, 2010; Bloomfield et al., 2019a; van der Wiel et al.

2019). In an optimal situation, wind and solar capacities might be adjusted to maintain balance between electricity demand and energy production at all time-scales (von Bremen, 2010). However, even with theoretically adequate installed capacities of wind and solar, the variability of RES and of demand, which 
is highly dependent on temperature, could result in periods of positive residual load (hereinafter referred to as RL), during which the production exceeds the demand, or in periods of negative RL with a surplus of RES generation (Engeland et al., 2017).

The effects of RES variability and the strong dependence on weather conditions have become the subject of recent studies that examine periods of low production by RES (Rife et al., 2016, Raynaud et al., 2018). In particular, extended periods of low production of wind, which have been termed dunkelflauten (from the German dunkel:absence of light and Flaute: absence of Wind)(e.g. EEA (2017), 2017; Ohlendorf and Schill, 2020), can be challenging during demand peaks (EEA (2017), 2017). While previous studies have examined the

40 fluctuations of wind power linked to the large-scale atmospheric circulation over Europe (e.g Grams et al., 2017; Weber et al., 2019), emerging literature focuses on the analysis of prolonged periods of low RES production. Raynaud et al. (2018) characterised periods of low production from RES, referred to as "energy production droughts", which were identified as consecutive days of energy production below a fixed threshold for each RES (i.e. wind, solar and hydropower). Similarly, they identified "energy supply droughts" as periods of high residual load. They found a large variability in the energy droughts between renewable sources and the considered European regions, and showed a large decrease in the frequency and duration of the energy droughts with a power system using a mix so of energy sources, rather than relying on just renewable generation. Following this approach, Jurasz et al. (2021) assessed the complementarity between solar and wind power in Poland, where energy droughts of wind power generation were more frequent than those of solar resources. This study also highlighted that the presence of local hybrid energy production systems (such as solar and wind) would reduce the variability and intermittency of the energy renewable production. Ohlendorf and Schill (2020) quantified the occurrence of low onshore wind power in Germany using 40 years of reanalysis data. They found that low-wind-power events are less frequent in winter than in summer, but that the maximum duration was evenly distributed throughout the year. 
Another recent study analysed the mismatch between energy supply and demand in California and the Western Interconnect (Rinaldi et al., 2021). The authors quantified the occurrence of energy droughts of renewable production when daily wind and solar power was less than half of the climatological mean. Recently, Brown et al. (2021) examined the climatology and synoptic conditions

65 linked to extreme reductions of wind and solar production at weekly time-scales over western North America. They identified a co-variability of wind droughts and higher than average solar power, due to the seasonal cycle and synoptic conditions, which highlights the need for energy-sharing resources (i.e. energy $\operatorname{mix})$.

Despite the increasing attention received by the so-called energy droughts in the recent literature, there is no clear established definition of energy supply droughts. Furthermore, quantitative frequency analysis on RES droughts are limited. Energy droughts (hereinafter referred to as ED) can be included within a multivariate framework in which their main characteristics, such as duration and severity, may be dependent on each other. Therefore, a bivariate frequency analysis is needed to express the dependence between the ED characteristics. Copulas have become very popular for multivariate frequency analyses (Zhang and Singh, 2019, Chen et al. 2013), as they allow for the joint simulation of different univariate distribution characteristics (e.g. duration and severity).

so Within the energy transition context, a comprehensive frequency analysis of ED is particularly important for evaluating the potential risks of power generation highly dependent on weather conditions. Thus, motivated by the successful application of copulas in meteorological and hydrological drought analyses, here we propose a bivariate copula-based approach to model the dependence between the two main features of the ED: duration and severity.

The remainder of this paper is organised as follows. Section 2 introduces the data used during this study. Also in Section 2, the energy-conversion models are summarised. Section 3 includes the ED definition and the description of the copula modelling procedure. The results are presented in Section 4 and Section эo 5 concludes our study with a general discussion and conclusions. 


\section{Data}

We use daily time series of hourly European electricity demand, solar and wind power at country level for 27 countries (Table S1). The data sets created by Bloomfield et al. (2019b) are a reconstruction of energy indicators (i.e. energy demand, wind and solar power), based on the ERA5 reanalysis product (Hersbach, 2018) that covers the period 1979-2019. The data set corresponds to one time series (at daily time step) per indicator and per country.

The data is available from the Reading Research and Data Repository ( http:dx.doi.org/10.17864/1947.227) and it has been used in previous studies (Bloomfield et al. 2019a, 2020). In the following section, we briefly summarise the methods used for the weather-to-energy conversion data. Interested readers are referred to Bloomfield et al. (2019a) for further details of the models construction and validation.

\subsection{Energy demand}

The electricity demand was reconstructed based on a multiple linear regression model trained with observed national demand, in giga $\left(10^{9}\right)$ watts (GW), corresponding to two complete years (2016-2017), extracted from the ENTSOe transparency platform (ENTSOE, 2019). The regression model uses both weather-dependent and human-behaviour-dependent predictors (e.g. the day-of-the-week and long-term socioeconomic trends, Bloomfield et al., 2019b). The weather-dependent model parameters are heating-degree days (HDDs) and cooling-degree days (CDDs). A HDD occurs when a country-average $2 \mathrm{~m}$ temperature falls below 15.5 degrees (the threshold at which residential heating is required) whereas a CDD occurs when a country-average $2 \mathrm{~m}$ temperature is 115 above 22 degrees and energy is required for residential cooling. Within the model $2 \mathrm{~m}$ temperatures are the only weather-dependent variable that contributes to fluctuations in demand. This style of multiple-linear regression based modeling is common in the literature (e.g. Raynaud et al., 2018, Francois, 2016, Thornton et al. 2017). 
As we are mainly interested in the meteorological impacts, here we use the weather-dependent model version that neglects the human behavioral factors, as in Bloomfield et al. (2019a). Thus, in this weather-dependent model version the predictors representing human behaviour (e.g. the weekday and socioeconomic predictors) are neglected in order to highlight the weather dependence (further details can be found in Bloomfield et al., 2019a).

\subsection{Wind and solar power}

Wind power capacity factors were obtained from a physical model that uses bias-adjusted wind speeds (using the Global Wind Atlas as the 'truth') at an altitude of $100 \mathrm{~m}$ above ground from the ERA5 reanalysis (Bloomfield et al. 2019a). Calibrated wind speeds are then passed through a power-curve to convert to wind power capacity factors. Different power-curves are used for different grid cells of the underlying climate data set: three turbine classes are retained, Class 1, 2 and 3. The choice of the turbine class per grid cell is dependent on the long-term average wind power generation. The three different turbine curves allow the maximum potential to be extracted from each grid-cells wind speeds. Country-level wind power generation is calculated by weighting each grid box by the amount of wind power installed there (in the reference year 2017).

Solar power capacity factors were modelled following the empirical formulation of Evans and Florschuetz (1977), using $2 \mathrm{~m}$ temperature and incoming surface solar radiation as inputs. The solar power capacity factors were created at each grid point and then aggregated to national level assuming a uniform distribution of solar panels across the country (as at the time of model creation there was not available data on panel locations). Both wind and solar power datasets captured the overall behaviour of the national wind and solar power generation well (see Bloomfield et al. (2019a) and references therein for further details).

The capacity factors (expressed in \%) obtained from both wind and solar power models were used to calculate the daily national wind and solar power production, for which we used as the baseline the installed capacity of wind and 
solar corresponding to 2017 (for each country) (Bloomfield et al., 2019a, 2020).

\section{Methods}

\subsection{Energy droughts: event definition}

We begin our analysis by defining ED events using a threshold-level based approach. An ED of low production is identified as an episode or period of time during which the energy production of renewable sources (i.e. wind and solar) is below the 10th percentile of the total generation (e.g. production of wind plus solar, which will be referred to as LWS) over the period of study 1979-2019. Similarly, an ED of the residual load (i.e. demand minus wind and solar production, referred to as RL) occurs when the 90th percentile of the distribution of RL is exceeded the 90th percentile of the distribution of RL. Table 1 summarises the events definition.

\begin{tabular}{|l|l|l|}
\hline Abbreviation & Description & Threshold \\
\hline LWS & Low wind and solar production & $<10$ th \\
\hline RL & Residual Load (demand - wind and solar production) & $>90$ th \\
\hline
\end{tabular}

Table 1: Summary of the events definition used in the study.

While the main results will focus on episodes of LWS and RL, similar frequency analyses were also applied to the individual sources (see section 4.1). Energy droughts that are separated by at least two days are treated as independent events.Then, we estimate the main characteristics of ED: 1) the duration, $\mathrm{D}$ (days), which is defined as the consecutive days below (or above) the selected threshold values and 2) the severity, S (GW) defined as follows:

$$
S=\sum_{i=1}^{D} \frac{\left|\left(E_{i}-E_{t h}\right)\right|}{\sigma}
$$

where $E_{i}$ is the energy production or load for the days (D) during a particular event, $E_{t h}$ is the threshold value and $\sigma$ is the standard deviation of RL or LWS. 
ED across countries due to the large variability, in terms of RL and LW

As shown in previous studies (van der Wiel et al. 2019, Bloomfield et al. 2019a), peaks of energy demand and low production show a strong seasonal variability. Therefore, the frequency analyses presented here are performed for two extended seasons: winter (October-Mach, ONDJFM) and summer (AprilSeptember, AMJJAS). Please note that the ED are defined based on absolute threshold values (i.e. considering the entire distribution throughout the year), and we then perform the frequency analysis for both extended seasons.

\subsection{Copula analysis}

Copulas (Nelsen, 2006) are flexible tools to describe the joint behavior of multiple variables and have shown a high potential in frequency analysis, multivariate modeling, simulation and prediction (Maity, 2018). Recently, the use of copulas has rapidly grown to examine dependence structures that exist between complex inter-correlated variables in many different areas, including hydrology, climate science or energy applications (Bhatti and Do, 2019). This methodology has gained popularity in the context of drought analysis (Chen et al., 2013. Michele et al., 2013). In a bivariate case, a copula is a joint distribution function that characterises the dependence between two random variables independently from their marginal distribution functions (Nelsen, 2006). For two random variables $X$ and $Y$ with marginal distributions $F_{X}(\mathrm{x})=\mathrm{P}(X \leq \mathrm{x})$ and $F_{Y}(\mathrm{y})=$ $\mathrm{P}(Y \leq \mathrm{y})$ respectively, a copula function can be used to construct their joint cumulative distribution function as follows

$$
F_{X Y}(x, y)=C\left(F_{X}(x), F_{Y}(y)\right)
$$

where $C$ is the copula of the transformed random variables $U=F_{X}(\mathrm{X})$ and $V=F_{Y}(\mathrm{Y})$, with the marginals $U$ and $V$ being uniformly distributed on the interval $[0,1]$. According to Sklar's theorem, if the marginal distributions are continuous, then the copula function $C$ is unique (Slark, 1996). The main advantage of using copula functions is the flexibility to model the dependence 
between multiple variables with different univariate marginal distributions. Interested readers can refer to Nelsen (2006) and Zhang and Singh (2019) for more the duration and the severity of the ED production or residual load exceed a certain value. In this case, the joint probability of exceedances of both $D$ and $S$ over a fixed threshold is expressed as: 


$$
P(D>d, S>s)=1-F_{D}(d)-F_{S}(s)+C\left(F_{D}(d), F_{S}(s)\right)
$$

where $F_{D}$ and $F_{S}$ are the marginal distributions of duration and severity, of ED.

$$
T=\frac{\tau}{P(D>d, S>s)}
$$

where $\tau$ is the average inter-arrival time (in years) of successive ED (i.e. total number of years divided by the total number of ED) and $P$ is the joint probability derived from the copula analysis (eq. 3). This approach is commonly used to assess meteorological and hydrological droughts (Zhang and Singh, 2019). We consider three threshold values $(75 \mathrm{th}, 90 \mathrm{th}$, and 95th) to define several classes of ED, for which the $\mathrm{T}$ were estimated. Please note that the percentiles are defined locally, i.e. for each country and season, as the copulas are applied separately 245 for each case. Let $d_{q}$ and $s_{q}$ denote the q-th percentile of the marginal distribution of the duration and severity, respectively. Table 2 shows the classification

\begin{tabular}{|l|l|}
\hline Class of ED & Joint Probability \\
\hline Moderate & $\mathrm{P}\left(\mathrm{D}>d_{75 t h} \& \mathrm{~S}>s_{75 t h}\right)$ \\
\hline Severe & $\mathrm{P}\left(\mathrm{D}>d_{90 t h} \& \mathrm{~S}>s_{90 t h}\right)$ \\
\hline Extreme & $\mathrm{P}\left(\mathrm{D}>d_{95 t h} \& \mathrm{~S}>s_{95 t h}\right)$ \\
\hline
\end{tabular}

Table 2: Classification of ED according to different threshold levels of both duration (D) and severity $(\mathrm{S})$. 
We further examined empirically the joint RP by counting the number of events for which both S and D exceed the selected thresholds. The empirievents, which is less of an issue when a parametric copula approach is implemented (Zscheischler and Seneviratne, 2017).

\section{Results}

Before starting the bivariate copula analysis, we examined the main characteristics of the ED, in terms of frequency, duration and severity. In addition to the ED of LWS and RL, we further assessed the events of the individual sources (i.e. wind and solar) as well as the corresponding demand-net-invidual RES: demand-net-wind, defined as the demand minus wind power, and demand-netsolar, defined as the demand minus solar power. Thus, we begin this section by presenting the frequency analysis of the EDs, followed by the ED assessments based on the copula results.

\subsection{Frequency analysis of $E D$}

Figure 1 illustrates the total number of ED per year obtained for LWS and RL. The frequency of LWS ED is generally larger in winter (11.4 events per 265 year on average) than in summer (7.6 events per year on average). However, significant variability in the occurrence of LWS events exists across the countries. While in most countries, the occurrence of LWS decreases in summer, the number of LWS ED in summer is comparable or higher than in winter for a few countries (e.g. Poland, Norway, Sweden, Latvia). This is explained by the small amount of installed solar capacity in those countries, and therefore the events of LWS are mainly driven by wind speed, which is reflected in the large frequency of ED of wind in summer, as shown in Fig. 2 for the individual wind source. The strong seasonality of solar power generation explains the reduced number of events of solar generation in summer over Europe (Fig. 2). 


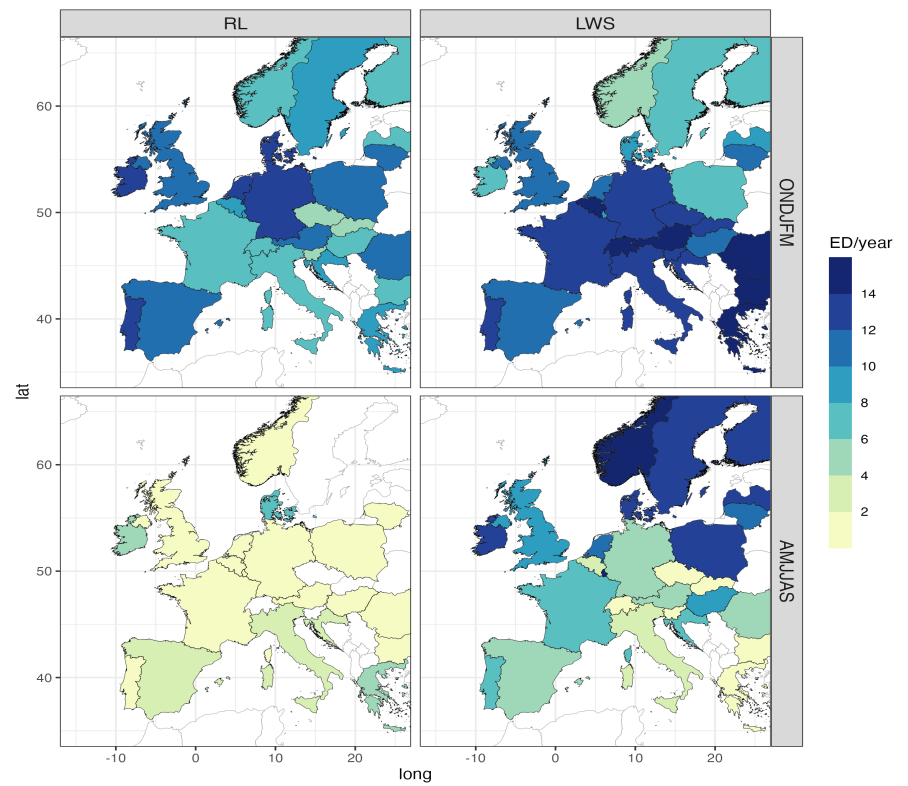

Figure 1: Number of ED per year of residual load (RL) and low wind and solar power (LWS) calculated for the extended summer (AMJJAS) and winter (ONDJFM) during the period of study 1979-2019 for each country. White areas within the countries of study indicate no ED. 


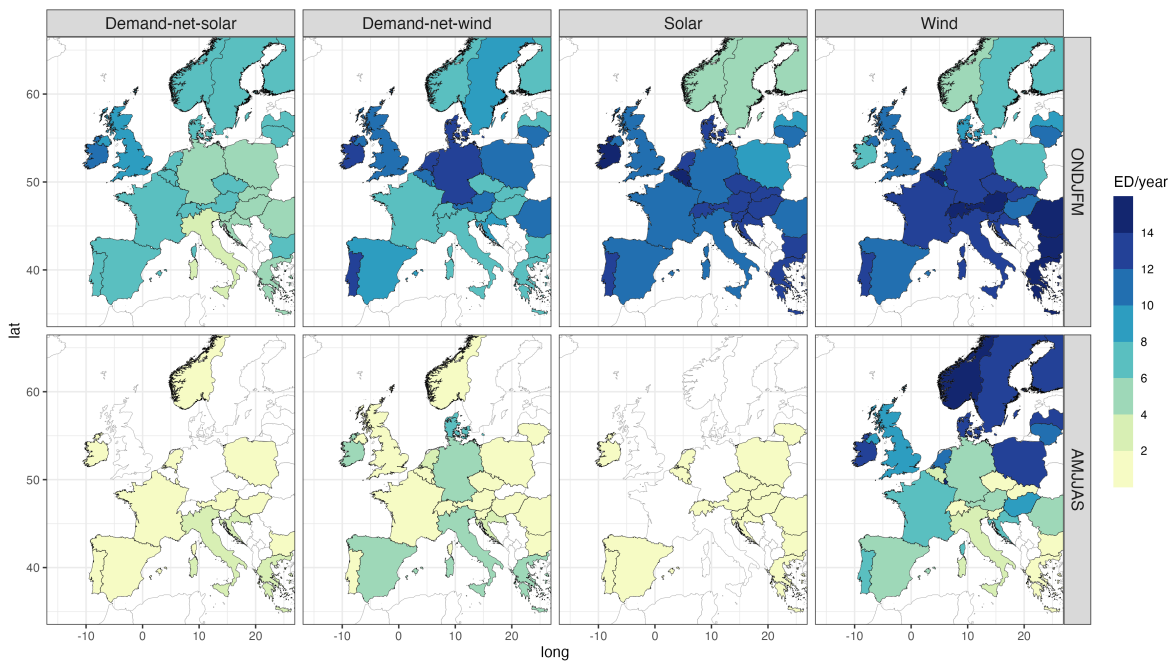

Figure 2: Number of ED per year of individual sources: demand-net-wind, demand-net-solar, wind power and solar power, calculated for the extended summer (AMJJAS) and winter (ONDJFM) during the period of study 1979-2019 for each country. White areas within the countries of study indicate no ED.

The lowest frequency of RL ED is observed in summer (1.6 events per year on average) due to the electricity demand patterns (i.e. low demand during the warmer months), and the number of RL ED is substantially higher in winter (11.4 events per year on average). Despite the reduced occurrence of RL ED in summer, a number of countries experienced more than $100 \mathrm{RL}$ events over the 41 year period. In the case of the northern countries, such as Denmark or Ireland, this can be attributed to the low amount of installed capacity of solar power, which lead to a high demand-net wind (see Fig. 2) that results in higher RL. The number of RL ED in the southern countries (e.g. Spain, Greece, Italy) is associated with the increasing use of air conditioning that results in demand peaks in summer (Bloomfield et al. 2019a; Thornton et al. 2017). Those countries also tend to experience the longest RL ED ( $\sim 15$ days) compared to the rest of the countries in summer (Fig. 3). Consistently with 
the seasonal patterns and with the weather dependence of both production and demand, overall, the maximum duration of RL ED and LWS ED is larger in winter (15.6 and 8.2 days) than in summer (4.8 and 5.6 days). It is worth to mention that the time series of RL generally show less variability compared to the timeseries of LWS. Moreover, as shown in Fig. 1, ED LWS are more frequent than ED RL, thus we could expect longer ED RL and shorter ED LWS. The mean duration ED also showed the seasonality of events, particularly in winter, when RL ED last on average 2.3 days compared to LWS ED that last 1.4 (not shown). Most European countries experience peak demands in winter, when the renewable production is also strongly influenced by seasonality (e.g. shorter daylight hours and reduced incoming solar radiation resulting in decreasing solar power generation) and weather patterns (e.g. persistent high-pressure systems associated with below normal wind speed that lead to decreasing wind power generation, Bloomfield et al. 2020 Raynaud et al. 2018). The ED of individual sources, more specifically the ED of solar and demand-net-solar are considerable shorter in summer than in winter due to their seasonality (i.e. more incoming surface radiation in summer) (Fig. 4). We highlight that ED could be of any length, but the longer duration events will provide the greatest challenges for energy system balancing. 


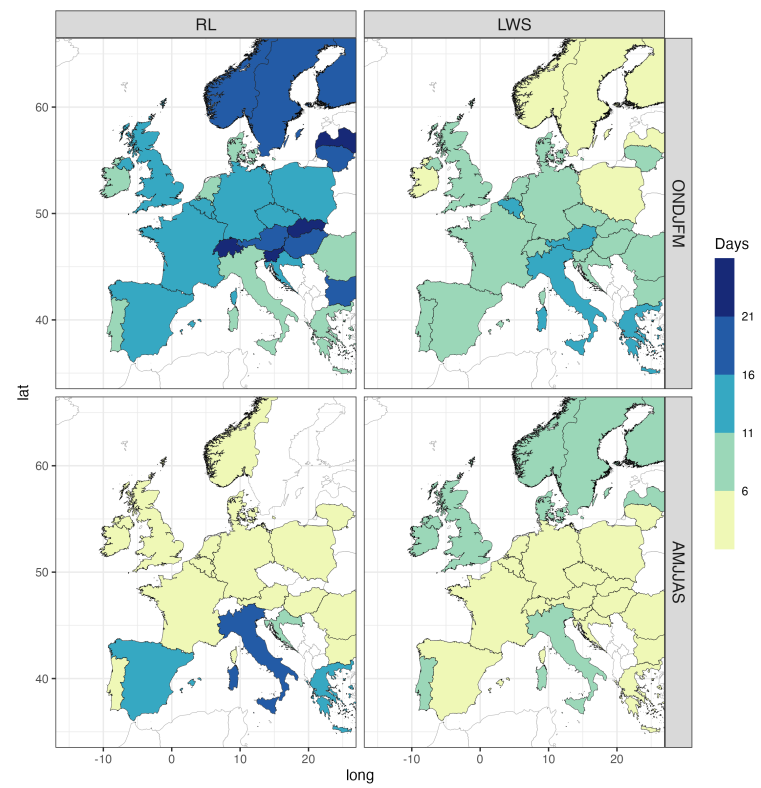

Figure 3: Maximum duration (days) of ED of residual load (RL) and low production wind and solar (LWS) calculated for the extended summer (AMJJAS) and winter (ONDJFM) during the period of study 1979-2019 for each country. White areas within the countries of study indicate no ED. 


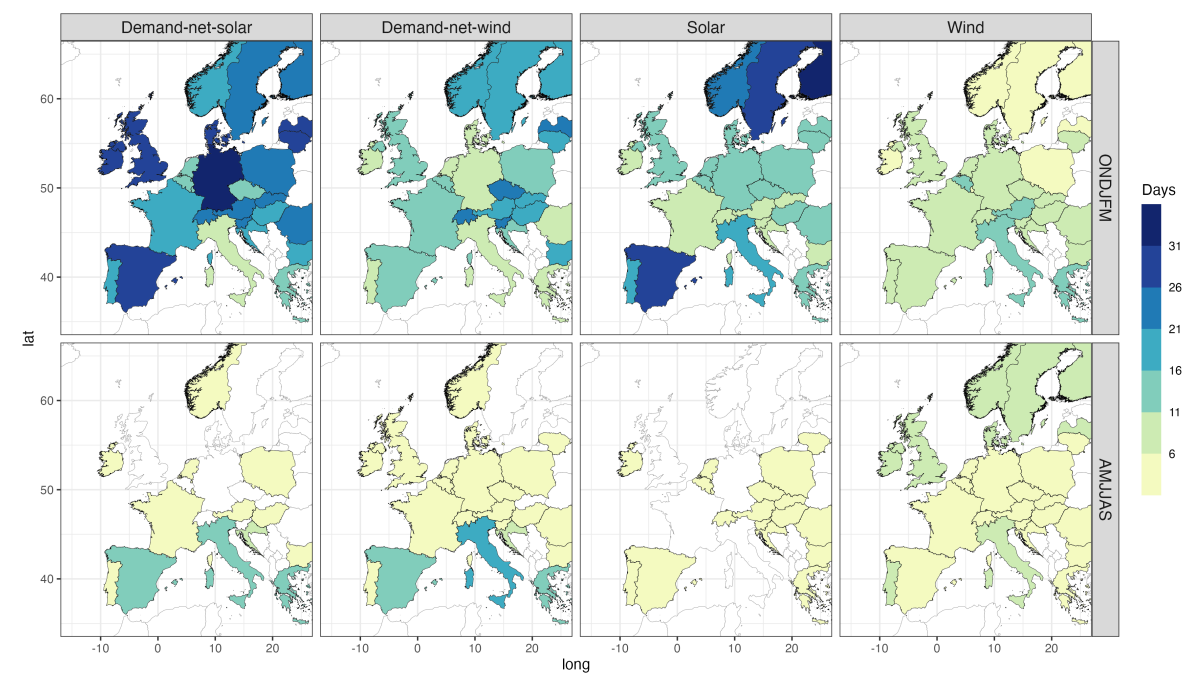

Figure 4: Maximum duration (days) of ED of individual sources: demand-net-wind, demandnet-solar,wind power and solar power, calculated for the extended summer (AMJJAS) and winter (ONDJFM) during the period of study 1979-2019 for each country. White areas within the countries of study indicate no ED.

The severity of both RL and LWS ED is higher in winter in most of the central and northern European countries (Fig. 5), which is consistent with more frequent and longer-lasting events in winter compared to summer (Fig. 1). Similar results are observed when examining the ED of individual sources, wind and solar, separately (Fig. 6). The severity of wind and solar ED is generally larger in winter, as well as the severity of the demand-net-wind and the demandnet-solar. Exceptions are found in the southern countries, such as Italy, where the most severe RL ED, in terms of both duration and severity, occur in summer. Also in Italy, it can be observed that the most severe demand-net-wind and the demand-net-solar events occur in summer. As stated above, this is explained by the higher summer demand peaks accompanied by low wind production, which result in high demand-net wind (i.e. demand minus wind generation, see Fig. 2). Moreover, in the case of Italy, the lower installed solar capacity (compared to 
other countries) explains more severe RL ED summer, in terms of duration and severity, which highlights the importance of the complementary sources within an energy mix, as shown in previous studies (Francois, 2016, Raynaud et al. 2018).

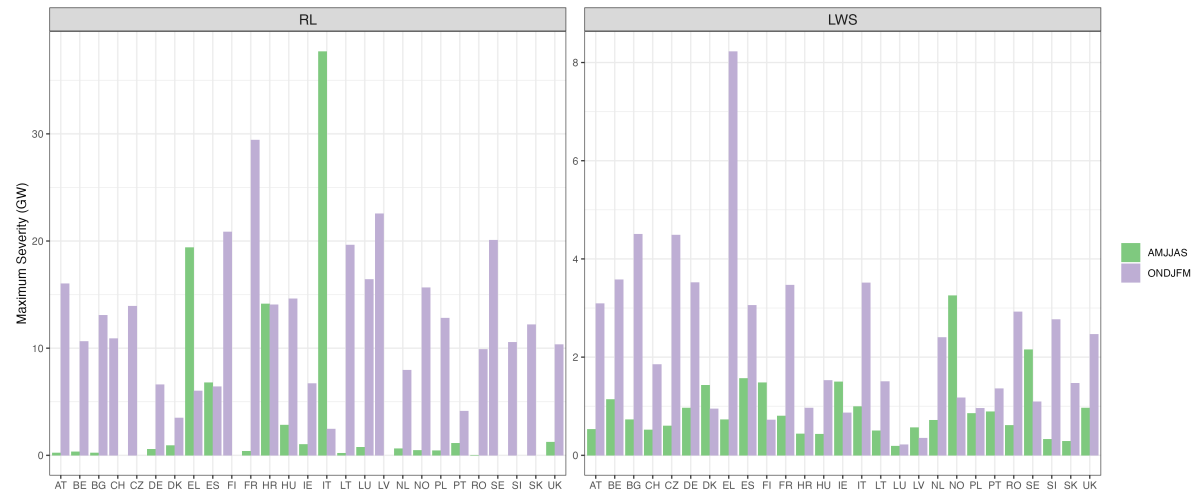

Figure 5: Severity of energy droughts (in GW) of RL (left) and LWS (right) for the extended summer (AMJJAS) and winter (ONDJFM) during the period of study 1979-2019 for each country. Please note the change of the scales due to the large variability between the RL and LWS. The long names of the country acronyms are displayed in table S1. 


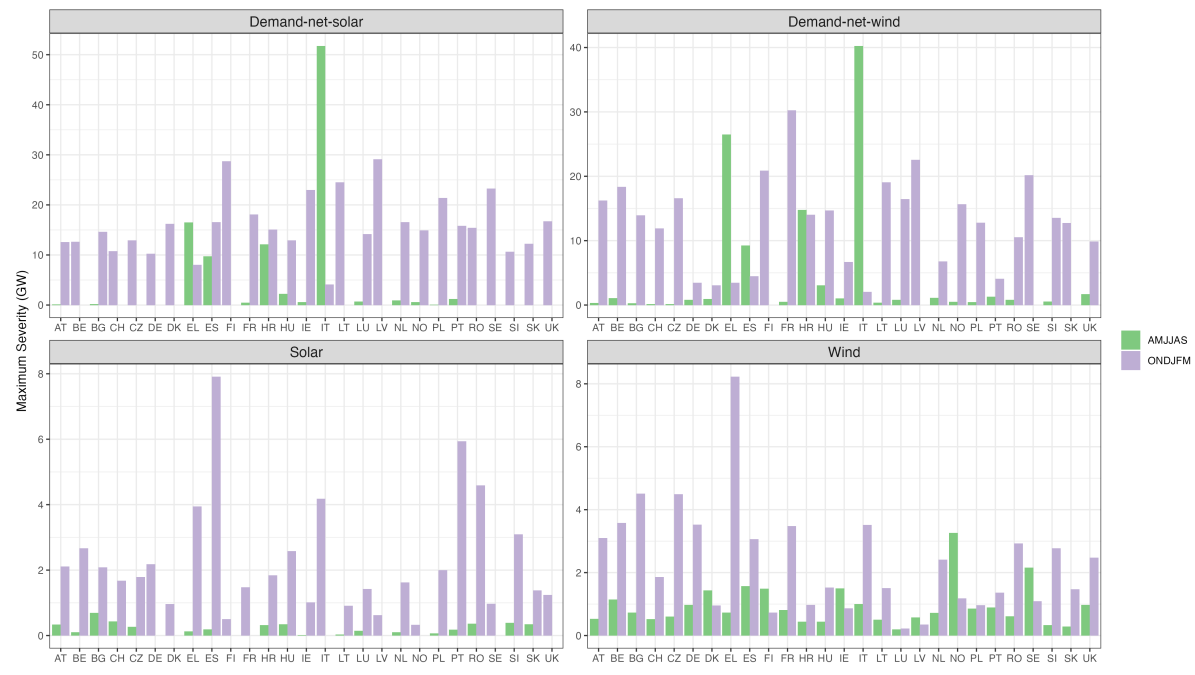

Figure 6: Severity of energy droughts (in GW) of individual sources: demand-net-wind and wind power, and demand-net-solar calculated for the extended summer (AMJJAS) and winter (ONDJFM) during the period of study 1979-2019 for each country. The long names of the countries acronyms are displayed in table S1. Please note the change of the scales due to the large variability between the demand-net quantities and the production.

\subsection{Bivariate return periods of ED}

An important step in the copula analysis is the fitting of the marginal distributions. Here, exponential and generalised extreme value (GEV) distributions are identified as the most appropriate to represent D and $\mathrm{S}$. The results from the copula selection process indicated that the Joe copula was best suited to capture the relationship between D and S in most cases (Tables S2, S3). The parameter experience high demand. Nevertheless, it can be noted that the dependence between D and S is particularly strong in summer in a few countries (e.g. Italy, 
Greece.), where the RL ED seem to be more severe than in winter, which is consistent with the frequency analysis presented above.

Given the dependence between the drought characteristics, the joint return periods are crucial to assess the potential risks associated to ED. Therefore, we calculated the joint return periods corresponding to three classes of ED (see Table 2). Furthermore, the empirical joint return periods were estimated directly from the number of observed events, and can be used to assess the robustness of the fitted copulas. Figure 7 shows the joint return periods for moderate LWS and RL ED, where a moderate ED is one for which both D and S simultaneously exceed the corresponding 75th percentile (derived from each seasonal distribution). As expected, moderate EDs are more frequent in winter than in summer. On average, the winter LWS ED and RL ED occur once every 0.58 years (i.e. $\sim 211$ days) and 0.67 years ( i.e. $\sim 246$ days), while in summer, LWS ED and RL ED occur every 2.4 years and 3.6 years (on average). It is worth noting that only a few countries are affected by moderate RL ED in summer (Fig. 5). These events tend to occur most frequently in countries such as Italy, Greece or Denmark, due to the summer peak demand or as a result of 355 a low production (e.g. low wind power generation in summer drives the LWS $\mathrm{ED}$, and thus, higher RL, in those countries with low solar installed capacities). Similar patterns of return periods were obtained when counting the number of moderate ED empirically(Fig.S3), which indicates a reasonably good agreement between both methods.

360

Severe LWS ED and RL ED (i..e exceeding the 90th percentile of the marginal distributions of D and S) are relatively frequent in winter ( on average, every $\sim$ 1.6 years) compared to summer (on average, every $\sim 8-9$ years)(Fig. 8). While in winter, the return periods are very similar across Europe, we found a large variability across countries in summer. For example, in some countries (e.g. Norway, the UK, Ireland) severe LWS ED occur very often, every 2-3 years, compared to countries that experience summer severe LWS ED less than once every 10 years. Such variability in summer return periods across countries was also found in the empirical return periods. However, we also noticed larger dif- 


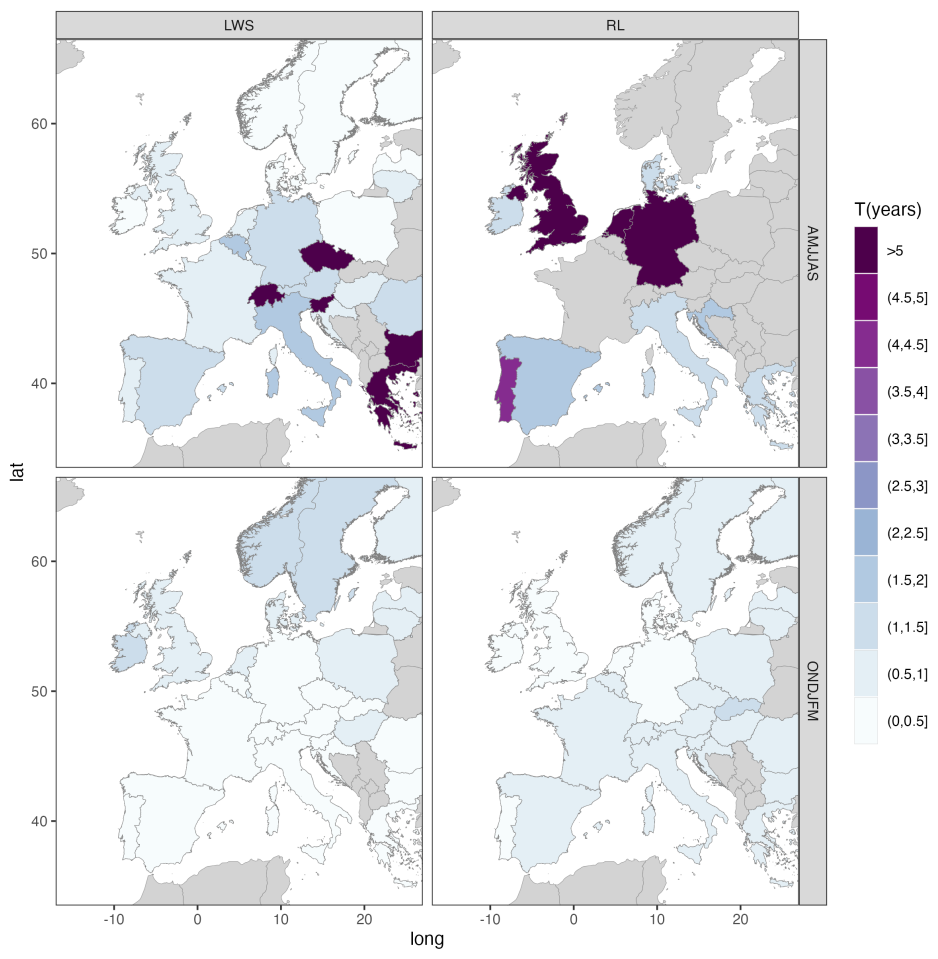

Figure 7: Joint return periods $(\mathrm{T})$ expressed in years corresponding to moderate ED (Table 2) of RL and LWS for both extended seasons, summer (AMJJAS) and winter (ONDJFM). Grey colors indicate countries where the copulas were not applied due to the limited number of ED.

ferences when comparing the empirical return periods and the return periods from the copulas than for the moderate ED in summer (Fig. S4). The differences are likely due to bias in the empirical estimates because the number of observed events is small.

As expected, the return periods increase with the severity of the ED, and extreme LWS ED and RL ED occur less often throughout the year (in Fig. 9). Similarly to moderate and severe ED, extreme ED are more frequent in winter. Overall, similar values were found for the different countries, with slightly larger return periods of ED of RL (every $~ 3.5$ years) than LWS ED, which tend to occur more often (every $\sim 2.9$ years). The variability across countries in 


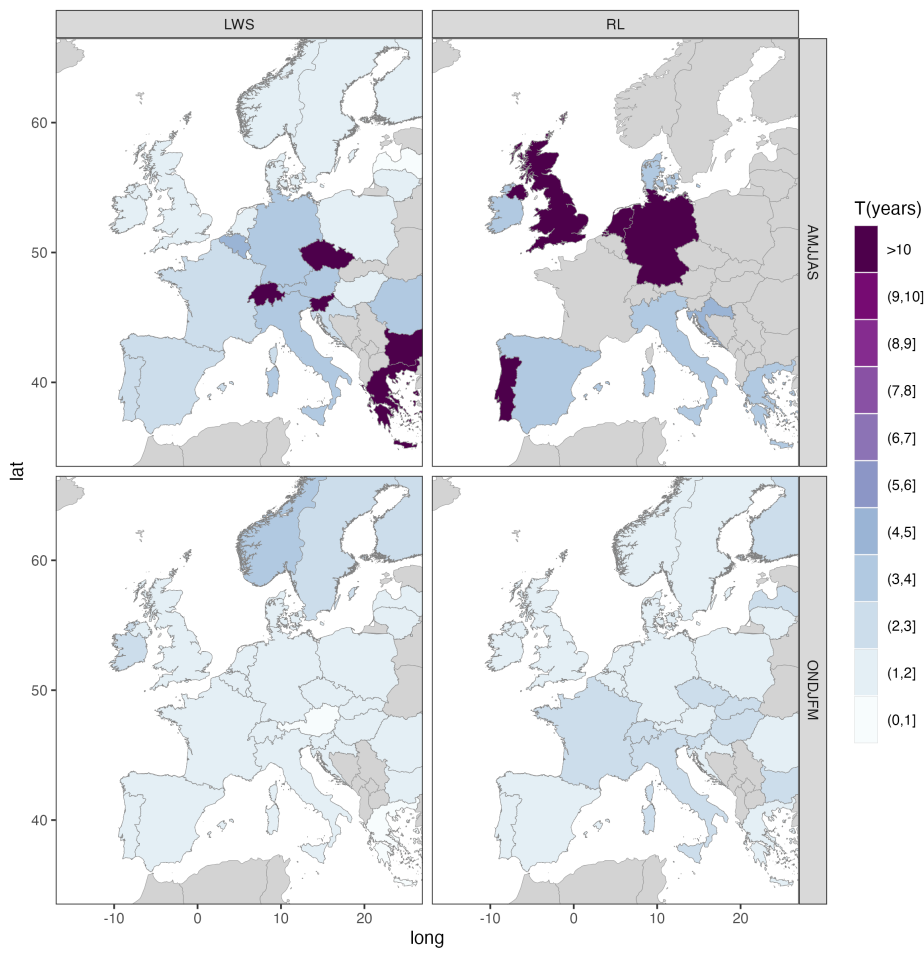

Figure 8: Joint return periods $(\mathrm{T})$ expressed in years corresponding to severe ED (Table 2) of RL and LWS for both extended seasons, summer (AMJJAS) and winter (ONDJFM). Grey colors indicate countries where the copulas were not applied due to the limited number of ED.

terms of return periods notably increases in summer, particularly in the case of LWS. Some countries (e.g. the UK, Denmark) seem to experience extreme ED of LWS quite often (every $\sim 2-4$ years), while in other countries (e.g. Switzerland, Poland) extreme LWS ED appear to be more rare (every $>30$ years). The empirical return periods obtained for winter show in general a good agreement with the return periods derived from the copulas (Fig. S5). Larger differences were found when comparing the summer return periods and in general, the empirical method underestimated the frequency of extreme ED (i.e. larger return periods). This might be explained by the fact that the empirical approach tend to show more limitations for rare events, as it might be the case with extreme ED (Tavakol et al., 2020, Zscheischler and Seneviratne, 2017). 


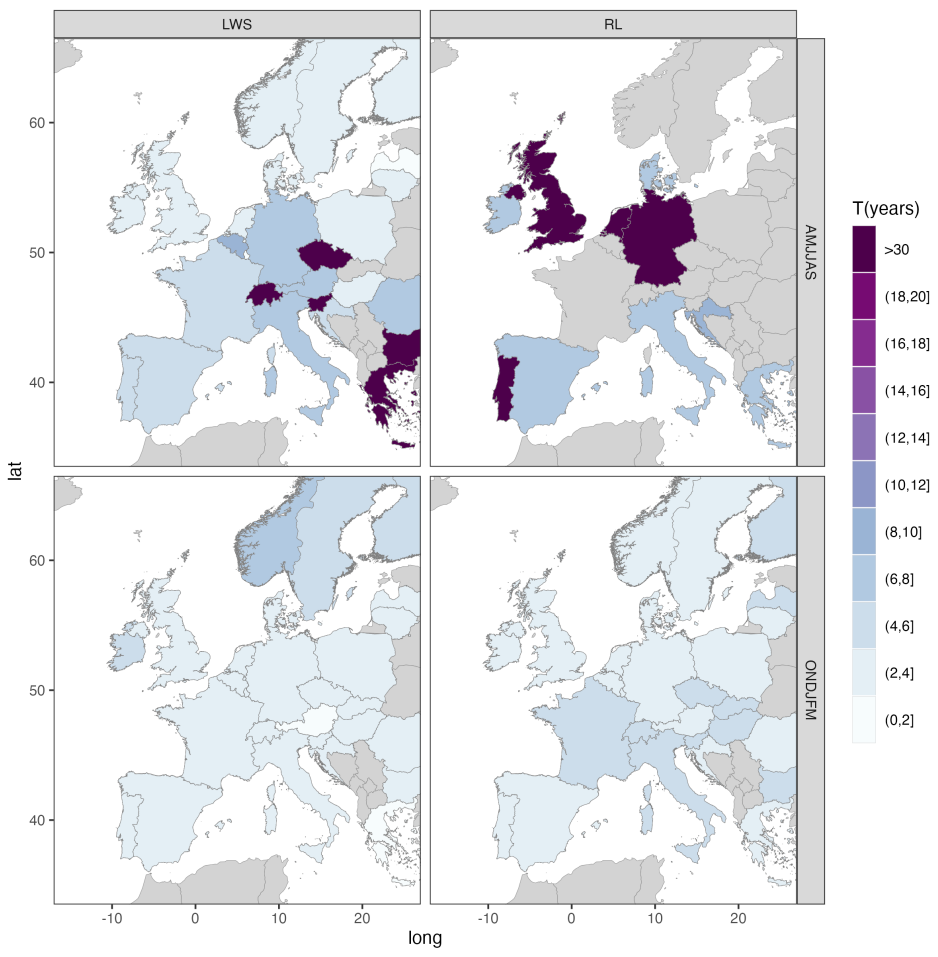

Figure 9: Joint return periods (T) expressed in years corresponding to extreme ED (Table 2) of RL and LWS for both extended seasons, summer (AMJJAS) and winter (ONDJFM). Grey colors indicate countries where the copulas were not applied due to the limited number of ED.

\section{Summary and Conclusions}

Characterizing periods of peak demand and periods of low power generation is crucial to address energy security concerns arising from the increasing share of renewable sources in the European energy supply. Renewable energy sources (RES), particularly wind and solar, are intermittent due to their strong weather dependence. Thus, the fluctuating power generation in periods of low production and high demand represents a major challenge for balancing energy supply and demand. Previous studies that analysed so-called energy droughts (e.g. Raynaud et al., 2018; Jurasz et al., 2021; Brown et al., 2021) suggested that the complementary behaviour of existing between wind and solar power would reduce periods of low energy production in systems with both renewable 
sources compared to systems with a single source.

A multivariate frequency analysis is essential to better understand the relationship between the characteristics (duration and severity) of the energy droughts, and thus to provide further insights into the severity of energy droughts ity demand, and wind and solar power generation, we examined energy droughts across 27 European countries for two extended seasons: winter (ONDJFM) and summer (AMJJAS). Energy droughts were identified based on a thresholdlevel definition and their duration and severity were estimated. We proposed the severity of periods of low production of wind and solar power (LWS) or high residual load $(\mathrm{RL})$. This multivariate approach enabled us to estimate the joint return periods to assess the risks of three types of energy droughts: i) moderate $\left(\right.$ duration $>d_{75 h} \&$ severity $\left.>s_{75 t h}\right)$, ii) severe $\left(\right.$ duration $>d_{90 t h} \&$ severity $>$ $\left.{ }_{415} s_{90 t h}\right)$, and iii) extreme (duration $>d_{95 t h} \&$ severity $>s_{95 t h}$ ). These percentiles are based on the observed distributions for duration and severity.

Given the strong weather-dependence of renewable sources (wind and solar), energy droughts of LWS and RL exhibited a marked seasonal pattern, being generally more frequent and longer lasting in winter than in summer. Compared 420 to winter, the number of summer energy droughts of RL is generally smaller across almost entire Europe, due to a general reduced electricity demand during the warmer months. However, exceptions are southern countries (e.g. Italy, Spain, Greece) that showed longer durations of residual load droughts in summer, which can most likely be explained by summer peak demand. Also in mark, Norway), as a result of episodes of low production of wind (which is the main contributor to the energy production here). This highlights the relevance of the complementaries between energy sources (Raynaud et al. 2018).

As expected, the most severe energy droughts occurred in winter for most 430 countries. Results from the frequency analysis for the individual power sources confirmed the strong seasonality of the demand-net-renewable (i.e. demand-net 
wind, defined as demand minus wind power generation, and demand-net solar, defined as demand minus solar power production) especially in the case of solar power, due to short daylight duration in winter, resulting in decreased solar 435 power production.

The dependence between the energy drought duration and severity was clearly reflected by the copula results that showed a stronger dependence in winter, particularly in the case of the residual load, for which the severity and the duration show a strong dependence for almost all countries. For each season, we analysed three energy droughts that were identified based on the exceedance of a threshold for both duration and severity. We showed that moderate energy droughts are very frequent in winter, with short return periods (e.g. moderate energy droughts of production occur every half year). The winter return periods of both production and residual load moderate droughts were similar across the countries, pointing out the strong relationship between the duration and the severity of droughts. Similar results were found for severe and extreme winter energy droughts although the return periods increase with the severity of the energy droughts. Our results pointed out smaller energy droughts in summer, especially from the load side, although we observed a large variability across 450 European countries.

In contrast to the previous studies that addressed the issue of energy droughts (e.g Raynaud et al., 2018; Ohlendorf and Schill, 2020; Jurasz et al., 2021), here we presented a multivariate frequency analysis in order to provide a better understanding of the energy droughts on the basis of the dependence structure of their main features: duration and severity. Our approach is similar to the copula-based assessments presented in the literature to analyse meteorological droughts by using well-known meteorological drought indices, such as standardized precipitation index or standardized precipitation evapotranspiration index) (e.g. Shiau, 2006, Zhang and Singh, 2019).

${ }_{460}$ For the time series of the power generation, we considered a baseline scenario of installed power capacity, in which we assume a total installed capacity corresponding to 2017. Accordingly, it must be acknowledged that using a different 
installed capacity might have an impact on the results, particularly in the case of residual load. While our analysis focuses on a current situation of installed

465 capacity, future research could explore the risk associated to energy droughts considering future power scenarios.

In summary, the multivariate copula-analysis used here provides new insights into the dependence structure of the main characteristics of energy droughts, namely duration and severity, which is crucial to estimate the potential risks of such events. The estimated joint return periods pointed out that in winter European countries are exposed to frequent (particularly moderate and severe) energy droughts of both production and residual load. In summer, there is an increasing variability across Europe and only a few countries experience frequent energy droughts. Overall, our results highlight the importance of the share of 475 renewable energy sources, which can greatly help to alleviate these prolonged episodes of energy droughts.

\section{References}

Bhatti M, Do RHQ. Recent development in copula and its applications to the energy, forestry and environmental sciences. Climate Dynamics 2019;44:1945373. doi/https://doi.org/10.1016/j.ijhydene.2019.06.015.

Bloomfield H, Brayshaw D, Charlton-Perez A. Characterizing the winter meteorological drivers of the European electricity system using targeted circulation

п types. Meteorolical Applications 2019a;27:e1858. doi:https://doi.org/10. $1002 /$ met. 1858

Bloomfield H, Brayshaw D, Charlton-Perez A. ERA5 derived time series of European country-aggregate electricity demand, wind power generation and q solar power generation. University of Reading 2019b;doi http:dx.doi.org/ $10.17864 / 1947.227$

Bloomfield H, Suitters C, Drew DR. Meteorological Drivers of European Power 

org $/ 10.1155 / 2020 / 548101$.

Brown P, Farnham D, K. Caldeira . Meteorology and climatology of historical weekly wind and solar power resource droughts over western North America in ERA5. SN Applied Sciences 2021;3:814.

Brucker T, Bashmakov I, Y. Mulugetta et al. . Climate change 2014: mitigation of climate change. Contribution of Working Group iii to the Fifth Assessment Report of the Intergovernmental Panel on Climate Change. Energy Systems 2014;:Cambridge University Press, Cambridge,United Kingdom and New York, NY, USA. by the trivariate plackett copula in the east river basin (China). Natural Hazards 2013;68:529-547. doi:https://doi.org/10.1007/s11069-013-0628-8

Cronin J, Anandarajah G, Dessens O. Climate change impacts on the energy system: a review of trends and gaps. Climatic Change 2018;151:79-93. doi:https://doi.org/10.1007/s10584-018-2265-4.

EEA (2017) . Renewable energy in Europe 2017 — recent growth and knock口 on effects. EEA Report 2017;3/2017. doi:https://www.eea.europa.eu/ publications/renewable-energy-ineurope-2017.

Engeland K, Borga M, Creutin JD, François B, Ramos M, Vidal JP. Space-time variability of climate variables and intermittent renewable electricity production - A review. Renewable and Sustainable Energy Reviews 2017;79:600-17. doi:https://doi.org/10.1016/j.rser.2017.05.046.

ENTSOE . ENTSOe transparency platform. European network of transa mission system operators for electricity data platform 2019;doi https:// transparency.entsoe.eu. 
European Climate Foundation . Roadmap 2050: a Practical Guide to a Prosperous, Low Carbon Europe. Brussels Available online, http://www roadmap2050eu/ 2010;.

Evans D, Florschuetz L. Cost studies on terrestrial photovoltaic power systems

with sunlight concentration. Solar Energy 1977;19:255-62. doi:https://doi. org/10.1016/0038-092X (77)90068-8

Francois B. Influence of winter North-Atlantic Oscillation on climate-related 미 energy penetration in europe. Renewable Energy 2016;:602e613doi 10.1016/ j.renene.2016.07.010.

525 Genest C, Rëmillard B, Beaudoin D. Goodness-of-fit tests for copulas: A review and a power study. Insurance Mathematics and Economics 2009;44:199-213. doi:https://doi.org/10.1016/j.insmatheco.2007.10.005

Grams C, Beerli R, Pfenninger R, Staffell I, Wernli H. Balancing Europe's wind-power output through spatial deployment informed by weather regimes.

530 \ Nature Climate Change 2017;7:557-62. doi/https://doi.org/10.1038/ nclimate 3338

Hersbach . Operational Global Reanalysis: Progress, Future Directions and Synergies with NWP. European Centre for Medium Range Weather Forecasts, Reading, UK 2018;18765. doi:10.21957/tkic6g3wm

${ }_{535}$ Jurasz J, Mikulik J, Dąbek P, Guezgouz M, Kaźmierczak B. Complementarity and 'Resource Droughts' of Solar and Wind Energy in Poland: An ERA5Based Analysis. Energies 2021;14:1118. doi $10.3390 /$ en14041118

Kiafar H, Babazadeh H, Sedghi H, Saremi A. Analyzing drought characteristics using copula-based genetic algorithm method. Arabian Journal of Geosciences 2020;13:745. doi https://doi.org/10.1007/s12517-020-05703-1.

Maity R. Statistical Methods in Hydrology and Hydroclimatology. Springer 2018;doi:https : //doi .org/10.1007/978-981-10-8779-0. 
Michele D, Salvadori G, Vezzoli G, Pecora S. Multivariate assessment of droughts: frequency analysis and dynamic return period. Water Resources Research 2013;49:6985-6994. doi:doi:10.1002/wrcr.20551.

Nelsen RB. An Introduction to Copulas. Springer Science and Business Media 2006 ;

Ohlendorf N, Schill W. Frequency and duration of low-wind-power events in Germany. Environmetal Research Letters 2020;15:084045. doi:https://doi. org/10.1088/1748-9326/ab91e9

Raynaud D, Hingray B, François B, Creutin J. Energy droughts from variable renewable energy sources in European climates. Renewable Energy 2018;125:578-89. doi:10.1016/j.renene.2018.02.130

Rife D, Cohan D, Collier J. A new kind of drought: U.S. record low windiness $555 \quad$ in 2015. Earthzine 2016;.

Rinaldi KZ, Dowling JA, Ruggles TH, Caldeira K, Lewis NS. Wind and Solar Resource Droughts in California Highlight the Benefits of Long-Term Storage and Integration with the Western Interconnect. Environmental Science \& Technology 2021;55(9):6214-26. doi 10.1021/acs.est.0c07848.

Sadegh M, Moftakhari H, Gupta H, Ragno HV, Mazdiyasni O, Sanders B, Matthew R, AghaKouchak A. Multi hazard scenarios for analysis of comq pound extreme events. Geophys Res Lett 2018;45:5470- 5480. doi:https: //doi.org/10.1029/2018GL077317.

Salvadori G, Durante F, Michelle CD, Bernardi M, Petrella L. A multivariate 565 copula-based framework for dealing with hazard scenarios and failure probabilities. Water Resources Research 2016;52:3701-3721.

Salvadori G, Michelle CD. Multivariate multiparameter extreme value models and return periods: a copula approach. Water Resources Research 2010;46:W10501. doi:10.1029/2009WR009040 
Shiau J. Fitting drought duration and severity with Two-Dimensional Copulas.

口 Water Resources Management 2006;20:795-815. doi/https://doi.org/10. 1007/s11269-005-9008-9

Slark A. Random variables, distribution functions, and copulas-A personal look backward and forward. distributions with fixed marginals and related topics.

575 Institute of Mathematical Statistics, Hayward, CA 1996;doi 10.1214/lnms/ 1215452606

Tavakol A, Rahmani V, Harrington Jr. J. Probability of compound climate extremes in a changing climate: A copula-based study of hot, dry, and windy events in the central United States. Environmental Reserach Letters 2020;15(10):104058. doi:10.1088/1748-9326/abb1ef

Thornton H, Scaife AA, Hoskins B, Brayshaw D. The relationship between wind power, electricity demand and winter weather patterns in Great Britain.

口 Environmental Research Letters 2017;12:064017. doi/https://doi.org/10. 1088/1748-9326/aa69c6.

${ }_{585}$ Tilloy A, Malamud B, Winter H, Joly-Laugel A. Evaluating the efficacy of bivariate extreme modelling approaches for multi-hazard scenarios. Natural

n Hazards and Earth Systems Sciences 2020;20:2091-2117. doi:https://doi. org/10.5194/nhess-20-2091-2020.

von Bremen L. Large-Scale Variability of Weather Dependent Renewable Energy Sources. In: Troccoli A, editor. Management of Weather and Climate Risk in the Energy Industry. Dordrecht: Springer Netherlands; 2010. p. 189206.

Weber J, Reyers M, Beck C, Timme M, Pinto J, Witthaut D, Schafer B. Wind Power Persistence Characterized by Superstatistics. Scientific Reports 2019;9:19971. doi https://doi.org/10.1038/s41598-019-56286-1.

Wenz L, Levermann A, Auffhammer M. North-south polarization of Eu- 
ropean electricity consumption under future warming. Scientific Reports 2017;114:E7910-E7918. doi/https://doi.org/10.1073/pnas.1704339114.

van der Wiel K, Stoop L, Zuijlen BV, Blackport R, den Broek MV, Selten F. Meteorological conditions leading to extreme low variable renewable energy production and extreme high energy shortfall. Renewable and Sustainable

口) Energy Reviews 2019;111:261-75. doi/https://doi.org/10.1016/j.rser. 2019.04 .065 .

Zhang L, Singh V. Copulas and their applications in water resources engi605 „ neering. Cambridge University Press 2019;doi/https://doi.org/10.1017/ 9781108565103

Zscheischler J, Seneviratne S. Dependence of drivers affects risks associated with compound event. Sci Adv 2017;3(6):1-11. doi 10.1126/sciadv.1700263 